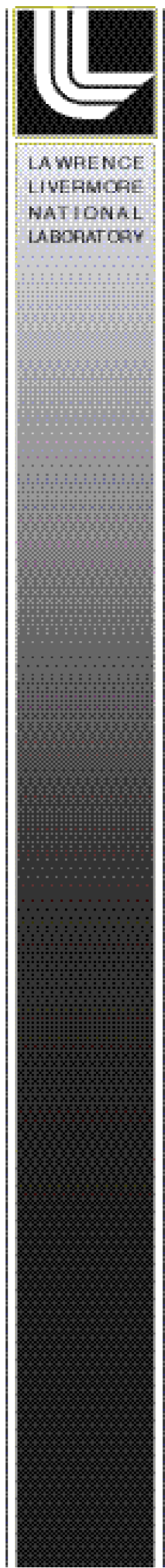

UCRL-TR-218946

\title{
Protein Classification Based on Analysis of Local Sequence-Structure Correspondence
}

A. T. Zemla

$02 / 13 / 2006$

This research is funded by the Laboratory Directed Research and Development (LDRD) Program at Lawrence Livermore National Laboratory (LLNL) under project 04-ERD-068. The LDRD Program is mandated by Congress to fund laboratoryinitiated, long-term research and development (R\&D) projects in support of the DOE and national laboratories' mission areas. The Director's Office LDRD Program at LLNL funds creative and innovative $R \& D$ to ensure the scientific vitality of the Laboratory in mission-related scientific disciplines. 
04-ERD-068

\section{Disclaimer}

This document was prepared as an account of work sponsored by an agency of the United States Government. Neither the United States Government nor the University of California nor any of their employees, makes any warranty, express or implied, or assumes any legal liability or responsibility for the accuracy, completeness, or usefulness of any information, apparatus, product, or process disclosed, or represents that its use would not infringe privately owned rights. Reference herein to any specific commercial product, process, or service by trade name, trademark, manufacturer, or otherwise, does not necessarily constitute or imply its endorsement, recommendation, or favoring by the United States Government or the University of California. The views and opinions of authors expressed herein do not necessarily state or reflect those of the United States Government or the University of California, and shall not be used for advertising or product endorsement purposes.

\section{Auspices Statement}

This work was performed under the auspices of the U.S. Department of Energy by University of California, Lawrence Livermore National Laboratory under Contract W-7405-Eng-48. 
04-ERD-068

\title{
FY05 LDRD Final Report
}

\section{Protein Classification Based on Analysis of Local Sequence- Structure Correspondence}

\section{LDRD Project Tracking Code: 04-ERD-068}

\author{
Adam Zemla, Principal Investigator
}

\section{Project Description}

The goal of this project was to develop an algorithm to detect and calculate common structural motifs in compared structures, and define a set of numerical criteria to be used for fully automated motif based protein structure classification. The Protein Data Bank (PDB) contains more than 33,000 experimentally solved protein structures, and the Structural Classification of Proteins (SCOP) database, a manual classification of these structures, cannot keep pace with the rapid growth of the PDB. In our approach called STRALCP (STRucture Alignment based Clustering of Proteins), we generate detailed information about global and local similarities between given set of structures, identify similar fragments that are conserved within analyzed proteins, and use these conserved regions (detected structural motifs) to classify proteins.

\section{Expected Results}

The software and database resulting from this project demonstrate how the problem of automation of the protein structure classification can be solved. Performed experiments demonstrate that our developed criteria and algorithm for automatic classification of proteins reflect the manual classification from SCOP database. Detected regions of structural conservation in analyzed proteins give us the ability to verify sequence-based alignments by comparison to the correctly calculated structural alignments. It significantly improves the quality of protein modeling, function recognition, and identification of regions on protein surfaces as candidates for ligand binding sites. Because accurate structural analysis is requisite to computational protein-based detection schemes, this work will improve the success rate and reduce the cost for choosing regions in proteins for antibody or high-affinity ligand recognition. It will also improve our ability to identify possibly cross-reactive proteins related to the protein targeted for detection. The part of our protein structure comparison and analysis system is already made accessible to the scientists through the web interface at http://as2ts.llnl.gov/AS2TS bringing positive recognition and visibility to LLNL and DOE.

\section{Mission Relevance}

The developed protein structure comparison system enhances the accuracy of protein classification and the quality of modeled protein structures. It implies the applicability for research related to the Laboratory's biodefense mission. This project leverages LLNL's capabilities in bioinformatics and high-speed computing; and the mission relevance to biodefense has been shown numerous times in FY04-FY05. Our developing STRALCP database of protein sequence-structure motifs used for protein structure classification improves protein modeling capabilities that enable us to predict more high-quality protein signature targets for pathogens of interest. The achieved capabilities of our system have been applied to improve structural models of critical proteins of causative agents of smallpox, ricin, plague, foot and mouth disease (FMD), monkeypox, and others. The generated models were used to predict the regions in protein structures upon which DNA and protein signatures designed at LLNL land, to determine potential unique protein signature candidates, to identify promising vaccine targets, and to suggest probable functions of unknown proteins. Exploitation of these models is ongoing at LLNL and by collaborators. 


\section{Accomplishments and Results}

This project builds on LLNL's capabilities in bioinformatics and high-speed computing, and enhances biodefense capabilities at the Laboratory by providing automated system of protein structure classification. We have developed a protein structure comparison algorithm to generate information about sequencestructure correspondence between related proteins. Our STRALCP system is capable to evaluate the level of overall structure similarity, and also to generate detailed information about the regions of local similarities between compared structures. We have designed a set of numerical criteria that use detected structurally conserved regions for automated protein structure classification. We have developed a prototype of protein structure database where proteins are clustered based on their similarity in identified structural motifs. Our automated clustering method detects relationships between proteins on the level of structural families with a very good agreement with manual SCOP classification. The developed automated structure classification capabilities will allow for better protein annotation of many microbes being studied in collaborative work with scientific groups from LLNL and other laboratories.

\section{Introduction}

There are many ways how a given set of protein structures could be clustered. Depends on the applied algorithm the results of the classification may differ significantly if different numerical criteria are used to assess the level of similarity between compared structures or if applied clustering criteria are focused on different features in protein structures. To perform particular clustering a suitable scoring function (or, in general, a scoring algorithm that takes into account a number of different features from compared proteins) has to be defined. Depending on the goal of the clustering it can be done by selecting one single measure or by combining different criteria to assess (score) the level of similarity between analyzed proteins. The goal of our research was to define criteria and to develop an algorithm for automatic classification of proteins that reflects the manual SCOP classification. The results from our research show that this goal can be achieved when the clustering is based on the combination of carefully selected different numerical criteria. In our approach, called STRALCP, we generate and combine detailed information about global and local similarities between any pair of analyzed protein structures, identify similar fragments that are conserved within analyzed proteins, and use such conserved regions to classify proteins according to their similarities in the detected structural motifs (spans). Our approach also allows automated detection of structural and sequence deviations within analyzed family or set of proteins.

\section{Results/Software Development}

(1) LGA_S structure similarity scoring function (overall similarity). LGA (Local and Global Alignment) program enables searching for the regions of local and global similarities and the "best" structure superposition between two protein structures. In order to consider local regions of the proteins in assessing their similarity a new scoring function has been implemented in LGA program. The LGA_S scoring function has two components, LCS (Longest Continuous Segments) and GDT (Global Distance Test), defined for the detection of regions of local and global structure similarities between analyzed structures (e.g. M-model and T-target). In comparing two protein structures, the LCS procedure is able to localize and superimpose the longest segments of residues that 
can fit under a selected RMSD (root mean square deviation) cutoff. The GDT algorithm is designed to complement evaluations made with LCS searching for the largest (not necessary continuous) set of "equivalent" residues that deviate by no more than a specified distance cutoff. Let:

$\mathrm{m}$ - the number of residues in $\mathrm{M}$ structure,

$\mathrm{t}$ - the number of residues in $\mathrm{T}$ structure,

$\mathrm{R}(\mathrm{v})=100 / \mathrm{t} * \mathrm{~L}(\mathrm{v})$, where $\mathrm{L}(\mathrm{v})$ is the length of the identified longest continuous segment of M:T residue pairs that fits under $\mathrm{v} \AA$ of RMSD cutoff,

$\mathrm{X}$ - the set of all M:T superpositions calculated by LGA algorithm, $\mathrm{G}(\mathrm{s}, \mathrm{v})$ - the number of M:T residue pairs for which the distance

between $\mathrm{Ca}$ (Carbon alpha) atoms is not greater than v Ångstroms after the superposition $\mathrm{s} \in \mathrm{X}$ is applied,

$D(v)=100 / t * \max \{G(s, v): s \in X\}$ is the maximal detected percentage of the $\mathrm{Ca}$ atoms in $\mathrm{T}$ structure that are within a distance threshold of $v \AA$ from

$\mathrm{M}$ structure upon calculated s superpositions,

LGA_S structure similarity scoring function is defined as a function of two structures $\mathrm{M}$ and $\mathrm{T}$ calculated as a combination of $\mathrm{R}(\mathrm{v})$ results from LCS calculations using set of $\mathrm{n}$ RMSD cutoffs $\mathrm{v}$ (e.g. $\mathrm{n}=3 ; \mathrm{v}=1.0,2.0,5.0)$, and $\mathrm{D}(\mathrm{v})$ results from GDT calculations using the set of $\mathrm{k}$ thresholds $\mathrm{v}$ (e.g. $\mathrm{k}=20 ; \mathrm{v}=0.5,1.0, \ldots, 10.0$ ):

where:

$$
\text { LGA_S }(M, T)=(1-w){ }^{*} S(\operatorname{LCS}(M, T))+w * S(G D T(M, T)),
$$

$$
\begin{aligned}
& S(\text { LCS })=\frac{2}{n \cdot(n+1)} \sum_{j=1}^{n}(n-j+1) * R\left(v_{j}\right), n=3, v_{j}=1.0,2.0,5.0, \\
& S(G D T)=\frac{2}{k \cdot(k+1)} \sum_{i=1}^{k}(k-i+1) * D\left(v_{i}\right), k=20, v_{i}=0.5,1.0, \ldots, 10.0,
\end{aligned}
$$

and $\mathrm{w}=0.75$ is a parameter $(0<=\mathrm{w}<=1)$ representing a weighting factor between LCS and GDT results.

The initial version of LGA program has been published in Nucleic Acids Research (A. Zemla: "LGA - a method for finding 3D similarities in protein structures", Nucleic Acids Research, Vol. 31, No. 13, 2003, pp. 3370-3374), and the Record of the Invention and the Patent Application has been submitted to Intellectual Property Law Group at LLNL.

(2) Detection of structurally conserved regions (similarity in the set of local regions). The cornerstone of our STRALCP algorithm is the ability to compare hundreds of protein structures in a single reference frame (target protein) and identify similar fragments that are conserved within a set of analyzed proteins. As an example of using our algorithm we show the results from the analysis of structure similarities between EAP domains from Staphylococcus Aureus (Eap2 (PDB entry: 1yn3), EapH1 (1yn4), EapH2 (1yn5)) and other proteins from PDB. On the plot below we show the set of PDB structures that were detected as most similar to EAP by our system. All identified by our algorithm proteins belong to one SCOP's superfamily called "Superantigen toxins, Cterminal domain". 


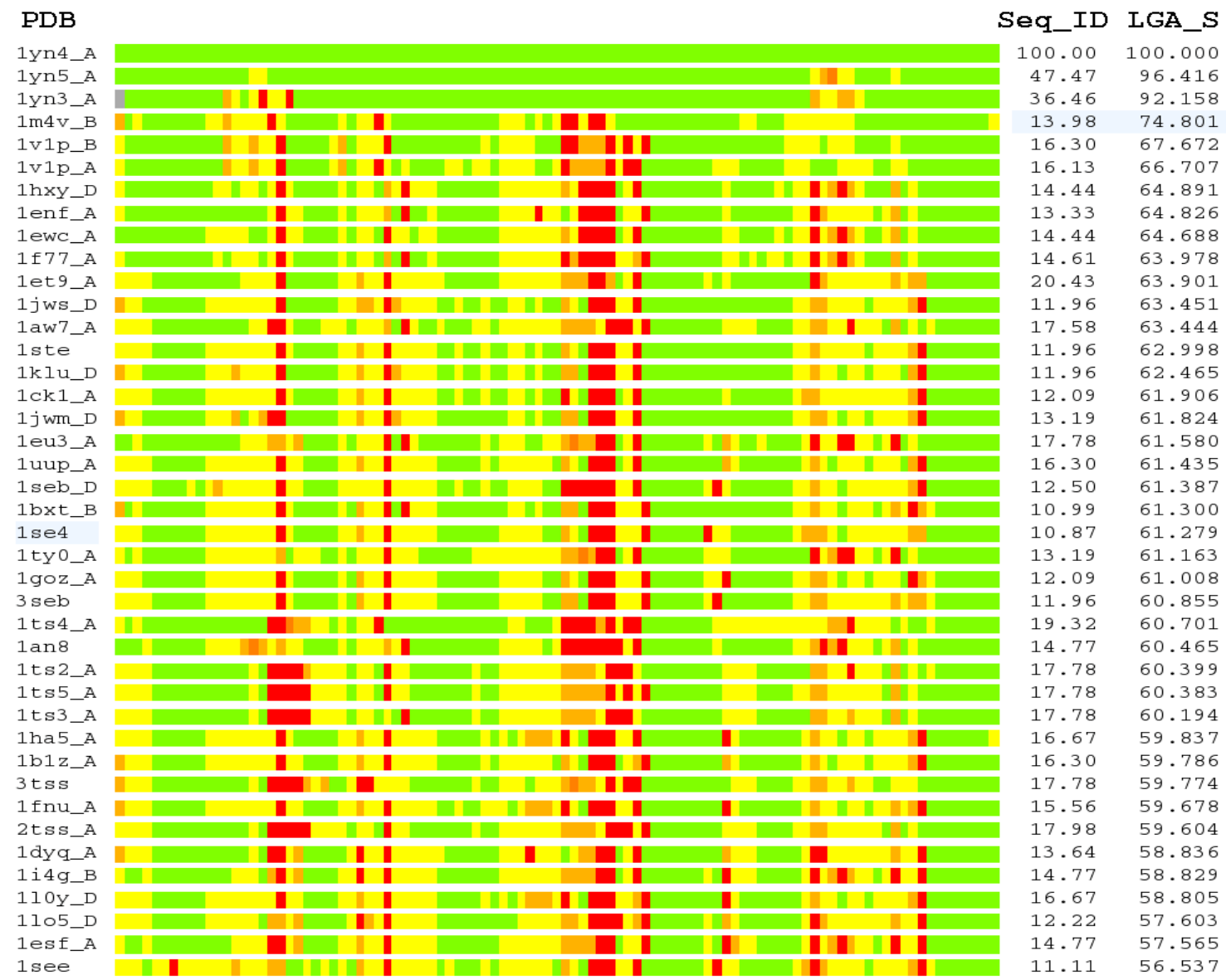

The plot above shows that all analyzed proteins are very similar (structurally conserved) in detected core regions (green). Colored bars represent Calpha - Calpha distance deviation between superimposed PDB structures and 1yn4_A (99 residues; from the left ( $\mathrm{N}$ terminal) to the right ( $\mathrm{C}$ terminal)). The distances between aligned residues are represented using different colors (from green - distances below $2 \AA$ to red - above $6 \AA$ ). The columns on the right side of the colored bars contain information about the level of sequence identity (Seq_ID), and level of calculated structure similarity (LGA_S). It is important to notice that the level of sequence identity (Seq ID column) between compared proteins is very low (below $20 \%$ ), so this classification was not possible to achieve by using sequence-based only techniques (e.g. PSI-BLAST analysis).

(3) STRALCP clustering algorithm. In our STRALCP approach we identify conserved regions and use them to classify proteins and protein domains according to their similarities in the detected structural motifs. For a given group of proteins and a target protein, the LGA algorithm creates one structural alignment of each protein to the target protein (see (2)). We cluster the proteins according to the structural regions they share with the target. Taking each of the proteins in turn as the target yields an ensemble of clusters, multiple partitions on the same set of proteins. Discrepancies are resolved by grouping together proteins that clustered together across many of the partitions. Each cluster of structures is defined by the representative structure and the set of shared structural features that we call a fingerprint. Comparison with the structural fingerprint determines whether a given structure belongs to the cluster. The clusters of proteins and 
the representative structures are created automatically. Below we show an example of using our algorithm where 1yn3-5 structures are clustered together (Cluster2) with four other protein structures: SET3 (PDB: 1m4v), SET1 (PDB: 1v1p), and TSST1 (PDB: law7, 2tss).

$\begin{array}{ll}\text { Cluster } & \text { Name } \\ \text { Cluster:1 } & \text { d1f77a2 } \\ \text { Cluster:1 } & \text { d1ck1a2 } \\ \text { Cluster:1 } & \text { d1goza2 } \\ \text { Cluster:1 } & \text { d1bxta2 } \\ \text { Cluster:1 } & \text { dluupa2 } \\ \text { Cluster:1 } & \text { d1fnua2 } \\ \text { Cluster:1 } & \text { dlesfa2 } \\ \text { Cluster:1 } & \text { d1ewca2 } \\ \text { Cluster:1 } & \text { d1ty0a2 } \\ \text { Cluster:1 } & \text { d1et9a2 } \\ \text { Cluster:1 } & \text { d1lo5d2 } \\ \text { Cluster:1 } & \text { d1sebd2 } \\ \text { Cluster:1 } & \text { d1hqrd2 } \\ \text { Cluster } & \text { Name } \\ \text { Cluster:2 } & \text { d1m4va2 } \\ \text { Cluster:2 } & \text { d2tssa2 } \\ \text { Cluster:2 } & \text { d1aw7a2 } \\ \text { Cluster:2 } & \text { d1v1pa2 } \\ \text { Cluster:2 } & \text { 1yn3_A } \\ \text { Cluster:2 } & \text { 1yn4_A } \\ \text { Cluster:2 } & \text { 1yn5_A }\end{array}$

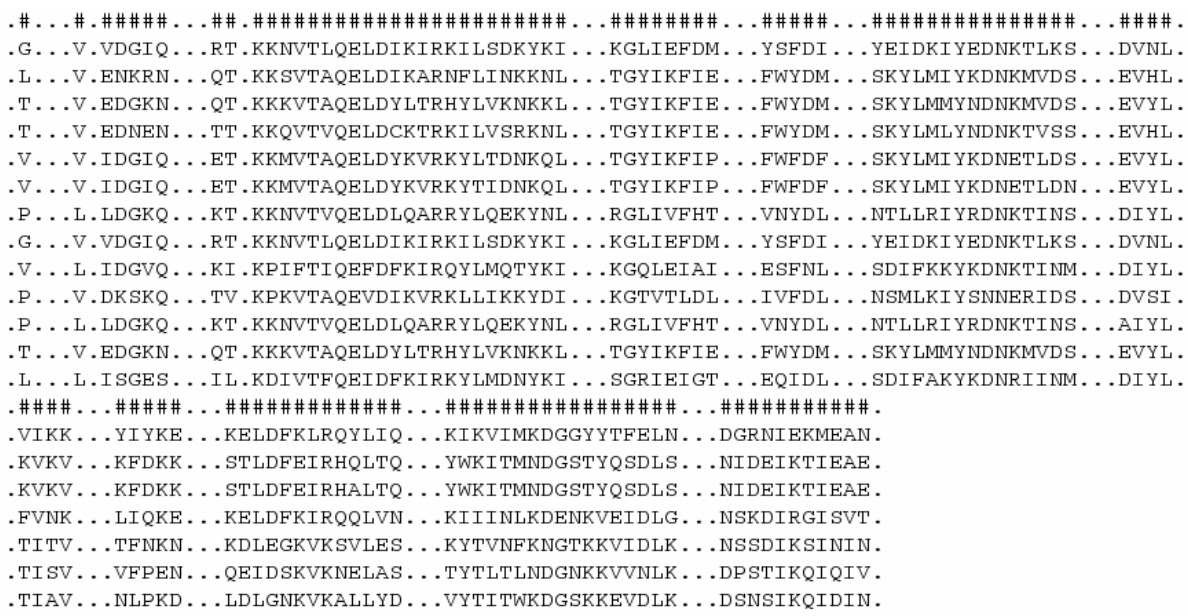

The biological functions of proteins 1yn3-5 are still unknown, but the results from calculated structure classification suggest close homology to four other structures from PDB. These results will guide future experimental work to help understand the functional role of these proteins.

Performed experiments. In our tests we have performed STRALCP calculations for over 50 SCOP families. The "quality" of the data deposited in PDB is comprised of protein structures having resolutions ranging from $0.54 \AA$ (X-ray crystallography) to greater than $15 \AA$ (electron microscopy). Despite this noise, the robust nature of our clustering method detects relationships on the level of the SCOP family with very good agreement with manually maintained SCOP classification. The experiments with clustering calculated using different single measures showed that our developed LGA_S scoring function gives much better result than traditionally used RMSD (root mean square deviation) value or $\mathrm{N}$ - number of residues superimposed under selected distance cutoff. For these comparisons we were using hierarchical clustering method from $\mathrm{R}$ package (version 2.1.1), a computer language and environment for statistical computing (see http://www.r-project.org/). In the example below we show the dendrogram generated for visualization of the hierarchical dependencies in the analyzed structures from the SCOP fold a.8 where LGA_S was used as a single measure for clustering. This example demonstrates the difference in two approaches: when a single measure is used to evaluate the overall level of structure similarity versus the multiple criteria based clustering (as implemented in STRALCP). 


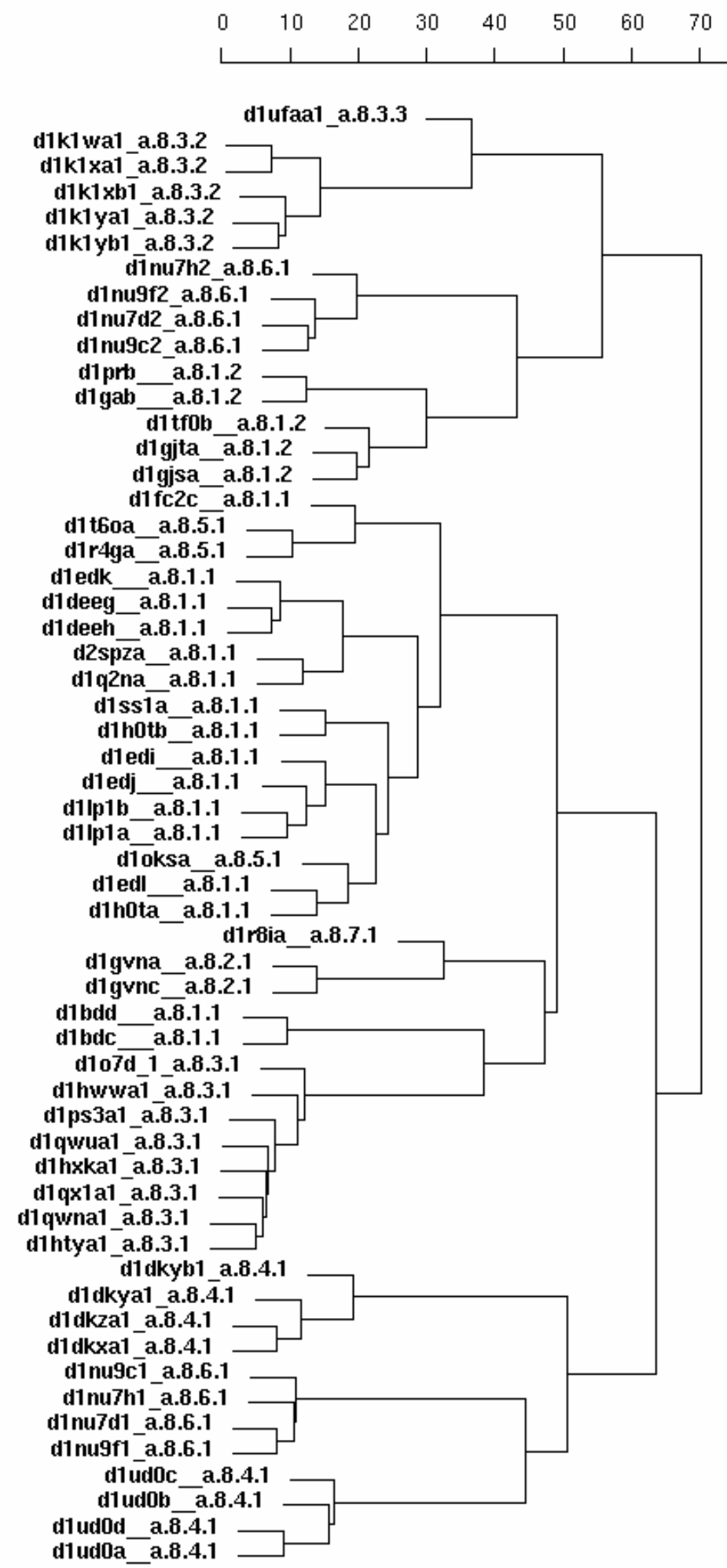

The dendrogram (to the left) shows the results of the LGA_S based clustering of SCOP entries from the fold a.8. Each code (entry_family) represents one protein from SCOP classification: entry and family number. On the right side the results of clustering created using our STRALCP approach are shown. This example shows that using STRALPC 
(multiple criteria based clustering) we can clearly separate proteins into appropriate clusters that correspond with a high accuracy to the SCOP families (see the last column to the right which gives SCOP family codes).

\section{Publication/Presentations}

Our developed protein structure analysis and modeling capabilities were used in collaborative work with many scientific groups in their biology research (see [1] - [8]). Some of the results from our research were presented on several conferences and workshops (see [9]- [14]).

\section{Papers:}

1) B. V. Geisbrecht, B. Y. Hamaoka, B. Perman, A. Zemla, D. J. Leahy: "Crystal Structures of Eap Domains from Staphylococcus Aureus Reveal an Unexpected Homology to Bacterial Superantigens", J.Biol.Chem, 2005, 280(17), pp. 17243-50. UCRL-JRNL-216376

2) J. B. Pesavento, M. Cosman, A. Zemla, P. T. Beernink, S. L. McCutchen-Maloney, J. P. Fitch, R. Balhorn, D. Barsky: "Identification of a thermo-regulated glutamine-binding protein from Yersinia pestis", Protein Science, (submitted), UCRL-JRNL-213767

3) R. Stanfield, A. Zemla, I.A. Wilson, and B. Rupp: "Antibody elbow angles are influenced by their light chain class", J.Mol.Biol., (in press), UCRL-JRNL-218128

4) P. J. Beuning, S. M. Simon, A. Zemla, D. Barsky, G. C. Walker: “A Non-Cleavable UmuD Variant that Acts as a UmuD' Mimic", J.Biol.Chem., (in press), UCRL-JRNL216587

5) C. Ecale Zhou, A. Zemla, D. Roe, M. Young, M. Lam, J. Schoeniger, R. Balhorn: "Computational approaches for identification of conserved/unique binding pockets in the A chain of ricin", Bioinformatics 2005 21: pp. 3089-3096. UCRL-JRNL-209388

6) A. Zemla, C. Ecale Zhou, T. Slezak, T. Kuczmarski, D. Rama, C. Torres, D. Sawicka, D. Barsky: "AS2TS system for protein structure modeling and analysis", Nucleic Acids Research, 2005, 33, pp. W111-W115. UCRL-JRNL-209684

7) S. D. Goens, S. Botero, A. Zemla, C. Ecale Zhou, M. Perdue: "Bovine enterovirus type 2. Complete genomic sequence and molecular modeling of the reference strain and a wild type isolate from endemically infected US cattle", Journal of General Virology, 85, 2004, pp. 3195-3203. UCRL-JRNL-202639

8) K. A. Kanterdjieff, Ch. Y. Kim, C. Naranjo, G. S. Waldo, T. P. Lekin, B. W. Segelke, A. Zemla, M. S. Park, T. C. Terwilliger, B. Rupp: "Mycobacterium tuberculosis RmlC epimerase (Rv3465): a promising drug-target structure in the rhamnose pathway", Acta Cryst., 2004, D60, pp. 895-902. UCRL-JRNL-202415 


\section{Presentations:}

9) A. Zemla, C. E. Zhou, M. Lam, J. Smith, B. Kirkpatrick. "A novel structure-driven approach for protein classification", a poster presented at the LLNL CAR Showcase Event, Nov. 3, 2005. UCRL-POST-216262

10) C. Zhou, M. Lam, J. Smith, A. Zemla, and T. Slezak. "Computational approaches for identification of targets for protein-based diagnostics" a poster presented at a meeting sponsored by the Dept. of Homeland

Security in Boston, April 26-28, 2005. UCRL-POST-211564

11) C. E. Zhou, A. Zemla, M. Lam, D. Roe. "Computational approaches for identification of signatures for protein-based diagnostics", a poster presented at a Gordon Conference in Buellton, CA, Jan 31 - Feb 4, 2005. UCRL-POST-209138

12) C. Zhou, M. Lam, A. Zemla, M. Yeh, T. Kuczmarski, C. Torres, J. Smith, T. Slezak, "Computational approaches to assay development for real-time detection of biothreat pathogens", a poster presented at a Keystone Symposium, Keystone, CO, Jan. 6-11, 2004. UCRL-POST-202649.

13) C. Zhou, A. Zemla, B. Vitalis T. Slezak. "Computational approaches for pathogen detection using protein-based signatures", a poster presented at the LLNL CBBB Media Event, Sept. 23, 2004. UCRL-POST-206450

14) C. Zhou, A. Zemla, T. Kuczmarski, M. Lam. "High-throughput selection of proteinbased signature targets for detection of bio-threat agents", a poster presented at an American Society for Microbiology conference on Functional Genomics and Bioinformatics Approaches to Infectious Disease Research, Portland OR, October 6-9, 2004. UCRL-POST-207543 